\title{
Artificial intelligence diagnosis of Helicobacter pylori infection using blue laser imaging-bright and linked color imaging: a single-center prospective study
}

\author{
Hirotaka Nakashimaa ${ }^{a}$ Hiroshi Kawahirab, Hiroshi Kawachic, Nobuhiro Sakaki ${ }^{a}$ \\ Foundation for Detection of Early Gastric Carcinoma, Tokyo; Chiba University; The Cancer Institute Hospital, \\ Japanese Foundation for Cancer Research, Tokyo, Japan
}

\section{Abstract}

\section{Introduction}

Esophagogastroduodenoscopy (EGD) is of growing importance in the diagnosis of Helicobacter pylori (H. pylori)

aFoundation for Detection of Early Gastric Carcinoma, Tokyo (Hirotaka Nakashima, Nobuhiro Sakaki); ${ }^{b}$ Center for Frontier Medical Engineering, Chiba University, Chiba (Hiroshi Kawahira); 'Department of Pathology, The Cancer Institute Hospital, Japanese Foundation for Cancer Research, Tokyo (Hiroshi Kawachi), Japan

Conflict of Interest: None

Correspondence to:

Hirotaka Nakashima, MD, Foundation for Detection of Early Gastric Carcinoma, 2-6-12 Nihombashikayabacho Chuo-ku, 103-0025 Tokyo, Japan, e-mail: nakashima@soiken.or.jp

Received 5 January 2018; accepted 26 March 2018; published online 3 May 2018

DOI: https://doi.org/10.20524/aog.2018.0269

(c) 2018 Hellenic Society of Gastroenterology gastritis because $H$. pylori infection is strongly associated with gastric carcinogenesis [1-4]. Studies have reported that conventional image-enhanced endoscopy (IEE) with a magnifying function is useful for improving diagnostic accuracy for $H$. pylori-associated gastritis [5-8]. However, since the light source was insufficient in conventional IEE systems, these systems were not able to diagnose a wide range of gastritis.

Recently, a new IEE system that uses a laser light source was introduced. This IEE system, called LASEREO (FUJIFILM Co., Japan), has two laser light sources, offering four observation modes of white light imaging (WLI), blue laser imaging (BLI), BLI-bright, and linked color imaging (LCI) [9-11]. This novel system appears appropriate for non-magnifying endoscopic diagnosis of $H$. pylori gastritis [11].

With progress in computer technology, artificial intelligence (AI) approaches are being increasingly applied in medicine. In particular, deep learning has attracted attention in diagnostic imaging $[12,13]$. Deep learning is a type of AI that imitates neural 
network functioning and can learn to identify scene-specific features in images; thus, it automatically establishes a classification protocol. Some previous articles reported the effectiveness of diagnosis of $H$. pylori infection using conventional endoscopic WLI with a deep learning method $[14,15]$. Nevertheless, there is no published article regarding the usefulness of new IEE systems without a magnifying function for the endoscopic diagnosis of $H$. pylori infection. Here, we attempted to generate an AI to diagnose $H$. pylori infection using BLI-bright and LCI without a magnifying function.

\section{Patients and methods}

\section{Study subjects and endoscopic examination}

We designed a prospective pilot study of all subjects who underwent EGD and were tested for serum $H$. pylori IgG antibodies at our medical clinic over a 13 month period beginning in November 2015. We obtained written agreement to participation in this study from all subjects. All of the 290 subjects were candidates for the study. Of these, 46 patients with a history of $H$. pylori eradication therapy were excluded, so as to avoid contamination of patients who had gastric intestinal metaplasia despite a negative reaction to serum H. pylori IgG antibodies. In addition, 22 subjects with serum $H$. pylori IgG antibody titers between 3.0 and
9.9 U/mL (so-called "high negative titer") were excluded to prevent the inclusion of false-positive or false-negative H. pylori infection in the study [16]. A serum H. pylori IgG antibody titer of $\geq 10 \mathrm{U} / \mathrm{mL}$ was considered positive for $H$. pylori infection, while a titer $<3.0 \mathrm{U} / \mathrm{mL}$ was considered negative. Finally, 222 subjects were stratified into those currently infected or uninfected with $H$. pylori. Fig. 1 shows the enrolment process for subjects in this study. The total cohort consisted of 139 males and 83 females, mean age $55.1 \pm 13.2$ years of age.

All EGDs were performed by an endoscopist $(\mathrm{HN})$, qualified as a board-certified fellow of the Japan Gastroenterological Endoscopy Society, blinded to the serum titer of H. pylori IgG antibodies. The endoscopic equipment used for the study was an EG-L580NW instrument (FUJIFILM Co., Japan). This endoscopic system has two laser light sources for excitation of WLI (typical wavelength: $450 \mathrm{~nm}$ ) and BLI (typical wavelength: $410 \mathrm{~nm}$ ), providing 4 different imaging observation modesWLI, BLI, BLI-bright, and LCI-by combining different emission strength ratios and image processing. BLI visualizes vascular microarchitecture and micro-surface feature of the gastrointestinal mucosa, similarly to narrow-band imaging [17]. LCI improves the ability of the endoscopist to recognize slight differences in mucosal color, such as those caused by inflammation or atrophy [11].

At our medical clinic, we routinely record approximately 40 WLI images of the stomach during EGD. In the present study, we focused on endoscopic images of the lesser curvature of the

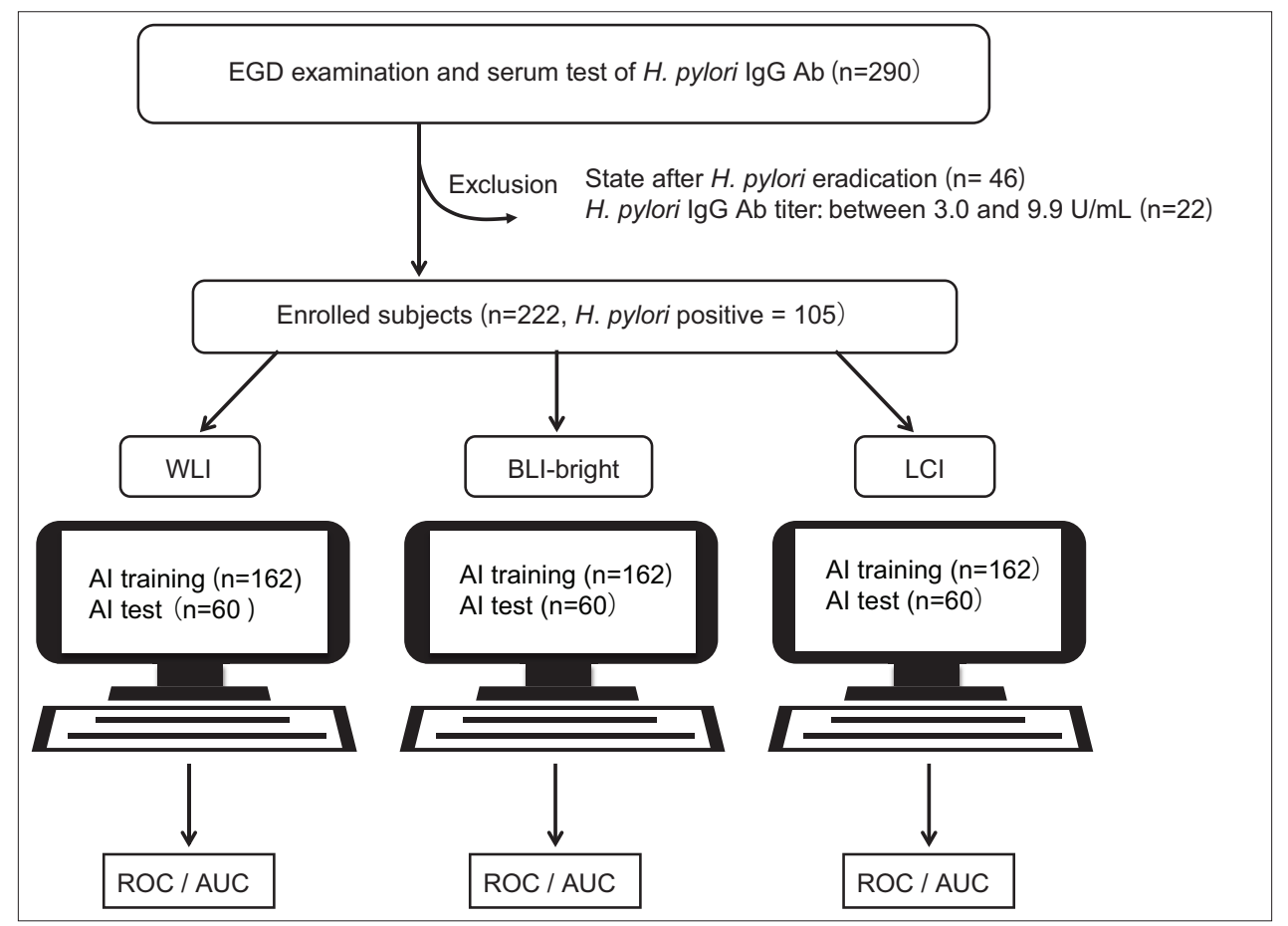

Figure 1 Enrollment of subjects in this study. Subjects who had a history of Helicobacter pylori (H. pylori) eradication therapy and a serum H. pylori IgG antibody titer between 3.0 and $9.9 \mathrm{U} / \mathrm{mL}$ were excluded. Finally, 222 subjects were enrolled (105 H. pylori-positive) who underwent esophagogastroduodenoscopy (EGD) and a serum test for $H$. pylori IgG antibodies. All of the 222 enrolled subjects were allocated to a training group ( $\mathrm{n}=162$ ) or a test group ( $\mathrm{n}=60$ ) WLI, white light imaging; BLI, blue laser imaging; LCI, linked color imaging; ROC, receiver operating characteristics; AUC, area under the curve; $A b$, antibodies 
gastric body, because mucosal features of atrophy and intestinal metaplasia are most prominent in this area $[18,19]$. During EGD, the endoscopist sequentially captured 3 still images at the same position in all subjects using WLI, BLI-bright, and LCI, with an endoscope fixed in position at the lesser curvature of the gastric body.

This study was designed according to the Helsinki Declaration of the World Medical Association and was approved by the ethics review committee of our medical foundation (approval number 15-02).

\section{Preparation of endoscopic images for Al}

The 222 enrolled subjects were allocated to a training group $(n=162)$ or a test group $(n=60)$ to evaluate the diagnostic accuracy of the AI. The subjects in the training group were registered in the first 10 months, and those in the test group were registered in the last 3 months of the study. The $H$. pylori IgG antibody titer of each subject was taken as the gold standard for infection status during evaluation of the AI diagnosis [20]. $H$. pylori infection was present in 75 of the training group and
30 of the test group subjects. For each patient, one WLI, one BLI-bright, and one LCI image of the lesser curvature of the gastric body was included.

For each image, a central part without personal information about the subject was clipped out at a resolution of $880 \times 880$ pixels as the region of interest and then converted to a 224 $\times 224$ pixel image to adapt to the visual calculation area of the AI. For training the AI, images linked to information on $H$. pylori infection status were employed as training images. For each training group (WLI, BLI-bright, and LCI) we prepared 486 images (rotated 90, 180, and 270 degrees) in addition to the original 162, for a total of 648. Thus, 1944 images were used for the AI training. After training, 180 images from the test group (60 each for WLI, BLI-bright, and LCI) were analyzed by the AI without information on $H$. pylori infection. The accuracy of the AI in diagnosing $H$. pylori infection was assessed by comparing the output data from the test group with the actual data on $H$. pylori infection status. In the study presented here, we used a total of 2124 endoscopic images for the training and test groups in a study design shown schematically in Fig. 2.

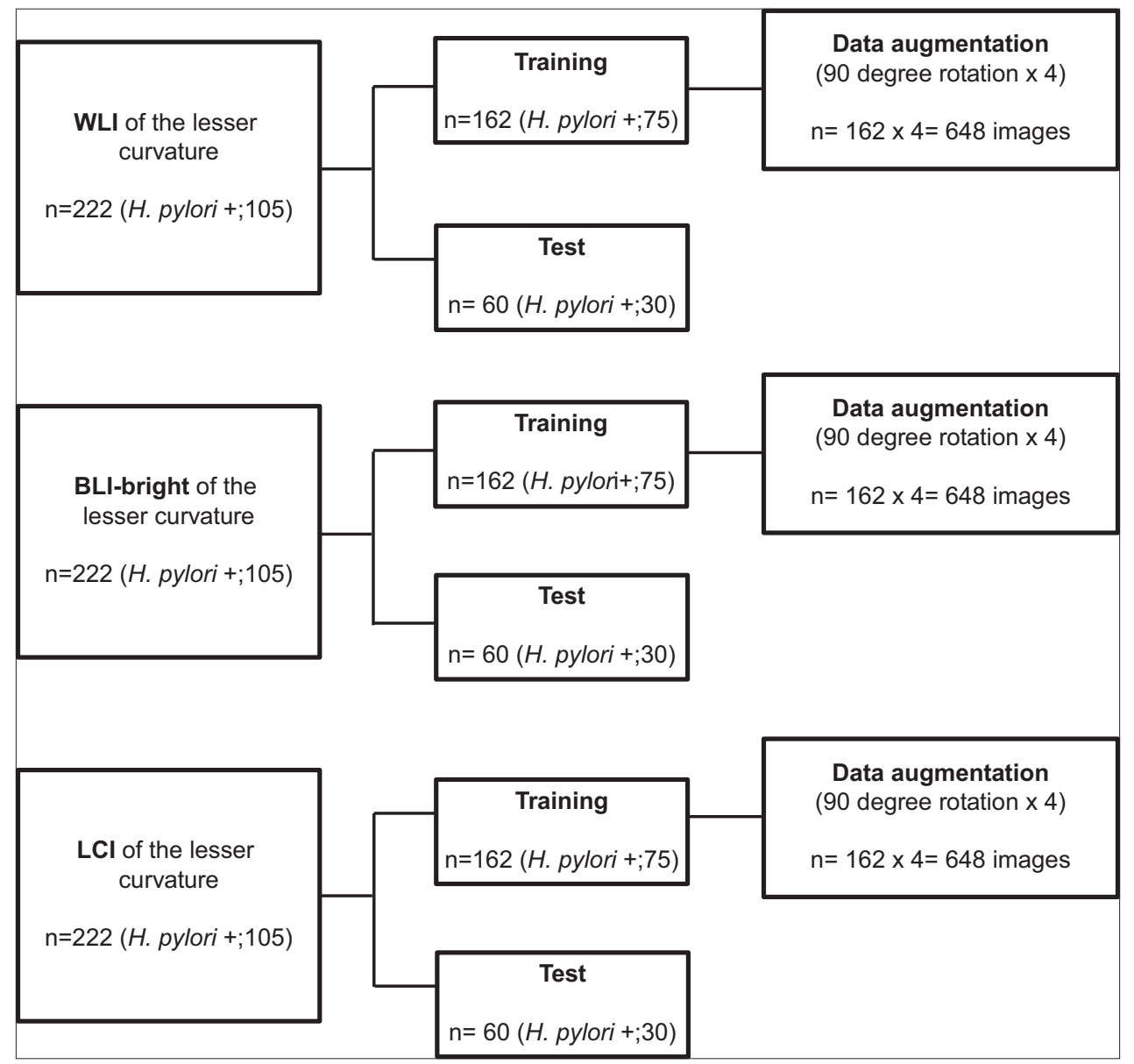

Figure 2 Data preparation for the study. In the present study a total of 2124 endoscopic images were used for the training and test groups. Data augmentation was applied to the training group but not to the test group

WLI, white light imaging; BLI, blue laser imaging; LCI, linked color imaging; H. pylori, Helicobacter pylori 


\section{The method of Al training and testing}

The specifications of the AI used in this study were as follows: operating system, Linux (Ubuntu 14.04, LTS Canonical Ltd. United Kingdom); neural network, GoogLeNet [21]; framework, Caffe (version 0.15.9) [22]; graphic processor unit, Geforce GTX TITAN X, (NVIDIA Co., USA). The GoogLeNet used was a 22-layer deep convolutional neural network (DCNN) incorporating convolutional and pooling layers.

Here, we elaborate on the AI utilized. We used GoogLeNet as a pre-trained DCNN model, created in advance using a large number of public supervised images. GoogLeNet sets its initial neural network weights based on 1.2 million general images [21]. In the present study, GoogLeNet was additionally trained with approximately 2000 endoscopic images from our training group, including data augmentation by image rotation, to diagnose the two categories sought $(H$. pylori-positive and H. pylori-negative). This method is called fine-tuning [23]. For the fine-tuning of the AI the batch size was set at 20, the training epoch at 100 and the initial learning rate at 0.0005 . The learning rate was stepped down by one digit after every third of the training epoch. The momentum was set at 0.9 , and the weight decay at 0.0005 . The AI automatically revised the original weights of the DCNN through fine-tuning, thus acquiring image recognition ability as an endoscopic diagnosis system.

\section{Statistical analysis}

The present study was an initial pilot study for endoscopic diagnosis using AI without sample size calculation. We determined the sample size, set at approximately 200 examinees, according to a previous study [24]. As noted above, a total of 180 test images obtained from 60 subjects $(30 \mathrm{H}$. pylori-positive and $30 \mathrm{H}$. pylori-negative images) were used for testing the AI. The diagnostic accuracy for $H$. pylori infection was determined quantitatively in terms of specificity, sensitivity, and receiver operating characteristic (ROC) area under the curve (AUC). The trained AI delivers a continuous predicted value between 0 and 1.0 for the probability of $H$. pylori infection corresponding to each test image. In this study, the predicted value for $H$. pylori infection yielded by the AI was used as a continuous variable for the ROC curve. The AUC was then determined comparatively for each WLI, BLI-bright and LCI case. All statistical analyses were performed using R computer software (version 3.3.2. The R Project for Statistical Computing, Austria) [25].

\section{Results}

Fig. 3 shows endoscopic images from a subject with $H$. pylori infection. In the WLI (Fig. 3A), the gastric mucosa of the lesser curvature was relatively smooth and yellowish. Fig. 3B shows an image in BLI-bright. There were small whitish spots scattered in the lesser curvature. In an LCI (Fig. 3C), there is a pale-white color change in the same area of mucosa. Fig. 3D,E,F are feature maps of convolutional layers during the AI test in WLI, BLI-bright and LCI, respectively. In each IEE image, the AI responded to the lesser curvature of the stomach, which was the region of mucosal atrophy with intestinal metaplasia and was indicated by a light green or a light blue color. The response of the AI to WLI (Fig. 3D) was relatively weak. In these 3 test images, the AI correctly diagnosed $H$. pylori infection.

Fig. 4 shows ROC curves, sensitivity, specificity, and AUC for WLI, BLI-bright, and LCI. The AUC was 0.66 for WLI, whereas for BLI-bright and LCI it was 0.96 and 0.95 , respectively. The AUCs were significantly larger for BLI-bright and LCI than for WLI (WLI and BLI-bright, $\mathrm{P}=3.37 \times 10^{-5}$; WLI and LCI, $\mathrm{P}=4.08$ $\left.\times 10^{-5}\right)$. The sensitivities for BLI-bright and LCI were $96.7 \%$ and $96.7 \%$, respectively. The diagnosis time for 60 test images by each AI was $7 \mathrm{sec}$. There was no difference in diagnosis time between WLI, BLI-bright, and LCI.

\section{Discussion}

IEE combined with magnifying endoscopy has been shown to be useful in the diagnosis of $H$. pylori-associated gastritis, because features of the image obtained using these endoscopic systems correspond well to the pathological findings of gastritis and mucosal atrophy [5-8]. Furthermore, the new laser IEE techniques of BLI-bright and LCI provide brighter endoscopic views and enable us to observe gastrointestinal mucosa over a wider area without a magnifying function, unlike conventional
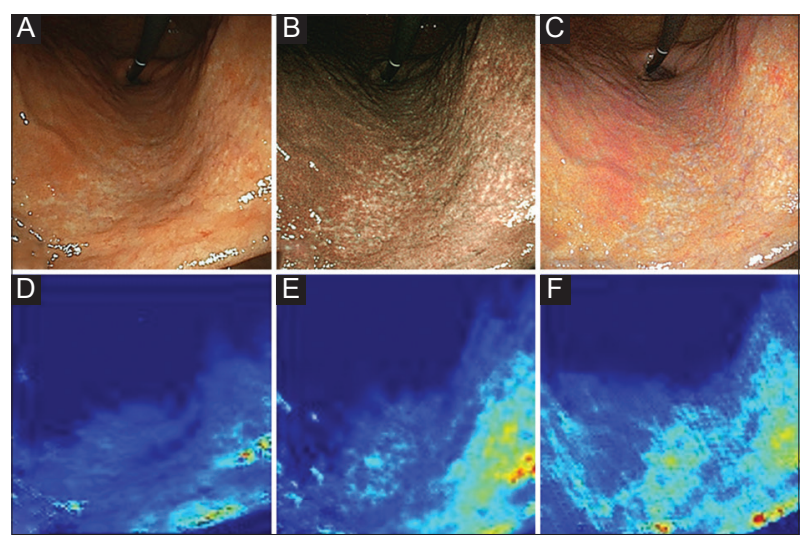

Figure 3 Feature maps of the AI corresponding to the endoscopic images. Endoscopic images of a $H$. pylori-positive subject (test group). An image in WLI of EGD (A) shows yellowish mucosa in the lesser curvature (lower part of the picture). An image in BLI-bright (B) shows small whitish spots scattered over the mucosal surface (region between the central part and the lower right part of the picture). An image in LCI (C) shows a pale-white color change in the same area. Feature maps of convolutional layers during the AI test are also shown for WLI (D), BLI-bright (E) and LCI (F). In each IEE image, the AI responded to the lesser curvature of the stomach, which was the region of mucosal atrophy with intestinal metaplasia, indicated by a light green or a light blue color

$A I$, artificial intelligence; WLI, white light imaging; BLI, blue laser imaging; LCI, linked color imaging; H. pylori, Helicobacter pylori 


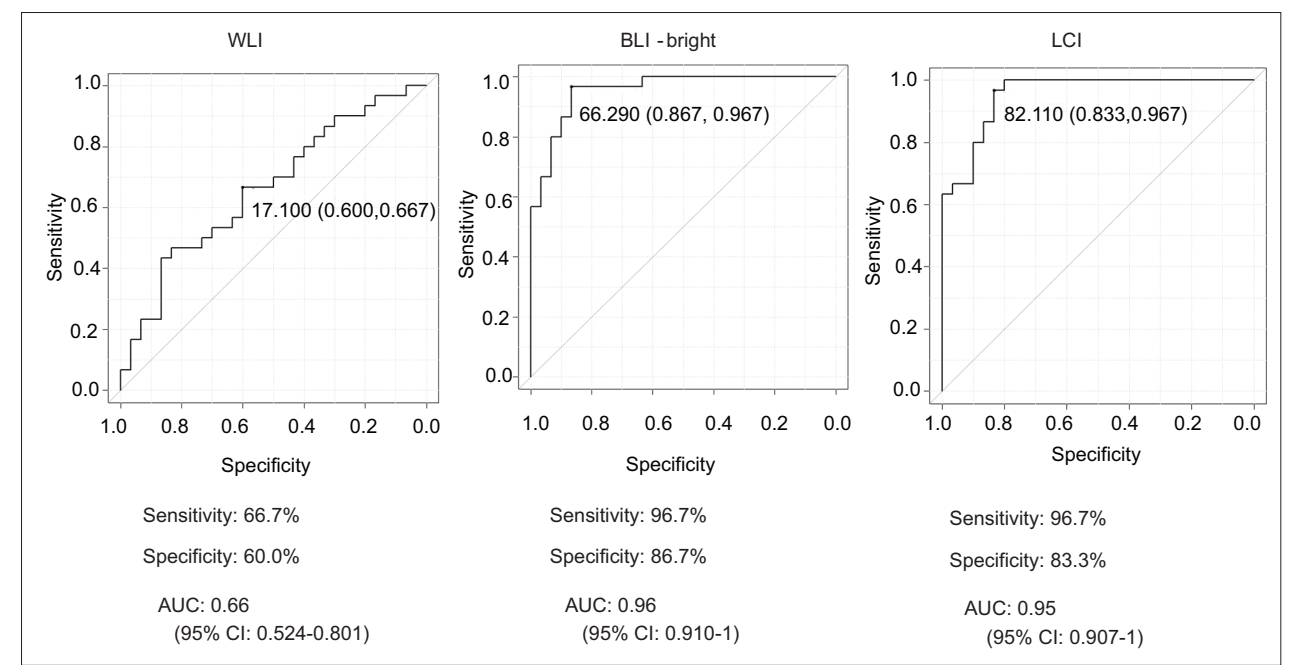

Figure 4 Sensitivity, specificity, and receiver operating characteristic curve of the AI. The sensitivity for BLI-bright and LCI was $96.7 \%$ and $96.7 \%$, respectively. The AUCs obtained for BLI-bright and LCI were significantly larger that for WLI (WLI and BLI-bright, P=3.37 $\times 10^{-5}$; WLI and LCI, $\mathrm{P}=4.08 \times 10^{-5}$ )

AI, artificial intelligence; WLI, white light imaging; BLI, blue laser imaging; LCI, linked color imaging; AUC, area under the curve 95\%CI, 95\% confidence interval

IEEs. Previous studies reported that the use of these new laser IEE systems facilitates the diagnosis of inflammation and atrophy at the mucosal surface [11]. However, there is no published evidence supporting the usefulness of new IEE systems without a magnifying function for the endoscopic diagnosis of $H$. pylori infection. Therefore, non-expert endoscopists may feel difficulty with the endoscopic image because a large amount of diagnostic information is contained in the picture with new IEEs [26].

Nowadays, progress in AI technology has resulted in its increasing application in medicine. In particular, deep learning has attracted attention in diagnostic imaging. Two articles have already reported on AI diagnosis systems for $H$. pylori infection; however, the previous AIs only used conventional white light endoscopic images in the diagnosis $[14,15]$. Itoh et al reported that their DCNN demonstrated an AUC and sensitivity for WLI of 0.956 and $86.7 \%$, respectively [14]. There were some environmental differences in DCNN between that paper and the present study, such as learning rate, training epoch, methods of data augmentation and the number of testing subjects. In this study, the authors mainly set the DCNN environment for adaptation to IEEs rather than WLI. Therefore, the sensitivity for BLI-bright and LCI was $10 \%$ superior to the previous paper using WLI. Shichijo et al described using a maximum of 32,208 endoscopic white light images to generate DCNNs; that is about 180 times more endoscopic images and training calculations than we used [15]. Other studies have evaluated the diagnostic accuracy of WLI and IEE for H. pylori gastritis. Anagnostopoulos et al reported that five expert endoscopists who performed over 2000 upper endoscopic examinations showed a positive predictive value of $83.8 \%$ for $H$. pylori infection using WLI with magnifying function [27]. Meanwhile, in the present situation, where IEEs are becoming more widespread, some endoscopists acknowledge that they can be effective for diagnosing gastritis [5-8]. Tahara et al reported a sensitivity of $95.2 \%$ and a specificity of $82.2 \%$ for magnifying narrow band imaging endoscopic findings in the prediction of $H$. pylori infection status [8]. Considering the prior reports and the current situation, we judged that AI could be created effectively using IEE. Hence, we aimed to generate a new AI for endoscopic diagnosis of $H$. pylori infection using two novel laser IEE systems: BLI-bright and LCI. Our new AI was efficiently adapted to those laser IEEs rather than WLI; consequently, it demonstrated an excellent ability to diagnose $H$. pylori infection using the IEEs. The AUCs for BLI-bright and LCI were 0.96 and 0.95, respectively; moreover, the sensitivity for BLI-bright and LCI was $96.7 \%$.

Our study had some limitations. First, the AI was constructed as a fine-tuned transfer learning model, it was not a fully developed program of DCNN for endoscopic diagnosis. Second, in this study we selected endoscopic images of the lesser curvature of the stomach for region of interest and excluded all patients who had a history of $H$. pylori eradication therapy. The reason is that our AI is not yet a mature enough tool to respond to the inflammatory findings. Endoscopic images of $H$. pylori-infected gastric mucosa show various features of inflammation, atrophy and intestinal metaplasia. An expert endoscopist recognizes such complex findings in the whole of the stomach, whereas our AI simply responds to the whitish atrophic mucosa with intestinal metaplasia in $H$. pylori-positive subjects and the smooth mucosa with regular arrangement of collecting venules in $H$. pylori-negative subjects solely in the lesser curvature of the stomach. There is a difference between an expert endoscopist and the AI in the diagnosis of $H$. pylori infection by EGD. In addition, our AI did not incorporate endoscopic images of other kinds of gastritis, such as autoimmune atrophic gastritis, environmental gastritis or bile gastritis $[28,29]$. Thus, we consider it is necessary to further improve the AI so that it can also respond to various inflammatory states in the $H$. pylori-infected mucosa and to 
avoid mistakes in diagnosing the other kinds of gastritis. If we can establish such a complete AI, it might be possible to demonstrate similar diagnostic accuracy for $H$. pylori infection or other gastritis in all regions of the stomach.

In the present study, we have generated an innovative image diagnosing system using AI and IEEs for diagnosing $H$. pylori infection. The authors believe that AI technology with IEE is likely to become a useful image diagnostic tool for EGD in the near future.

\section{Acknowledgments}

We wish to thank Mr. Takumi Itoh, Graduate School of Engineering, Chiba University, for technical support with deep learning. We also wish to thank Mr. Masahiro Kubo for advice on a laser endoscopy system.

\section{Summary Box}

\section{What is already known:}

- Esophagogastroduodenoscopy(EGD) is of growing importance in the diagnosis of Helicobacter pylori (H. pylori) gastritis, because $H$. pylori infection is strongly associated with gastric carcinogenesis

- Studies have reported that use of image-enhanced endoscopy (IEE) with magnifying function is useful for improving the diagnosis of $H$. pylori infection. However, a non-expert endoscopist may have difficulty evaluating this image information

- We have reported the effectiveness of diagnosis of $H$. pylori infection in endoscopic white light images using a deep learning method

What the new findings are:

- We aimed to generate a computer-aided endoscopic diagnosis system for $H$. pylori infection using IEE and artificial intelligence (AI) technology of deep learning

- The AI demonstrated an excellent ability to diagnose $H$. pylori infection using the novel IEEs

- AI technology with IEE is likely to become a useful image diagnostic tool for $H$. pylori infection

\section{References}

1. IARC Helicobacter pylori Working Group. Helicobacter pylori eradication as a strategy for preventing gastric cancer. IARC Working Group Reports, No. 8. Lyon, France: International Agency for Research on Cancer: 2014. Available from: https://www.iarc. fr/en/publications/pdfs-online/wrk/wrk8/Helicobacter_pylori_ Eradication.pdf

2. Sakaki N, Momma K, Egawa N, Yamada Y, Kan T, Ishiwata J. The influence of Helicobacter pylori infection on the progression of gastric mucosal atrophy and occurrence of gastric cancer. Eur J Gastroenterol Hepatol 1995;7 Suppl 1:S59-S62.

3. Uemura N, Okamoto S, Yamamoto S, et al. Helicobacter pylori infection and the development of gastric cancer. $N$ Engl $J$ Med 2001;345:784-789.

4. Kato T, Yagi N, Kamada T, Shimbo T, Watanabe H, Ida K; Study Group for Establishing Endoscopic Diagnosis of Chronic Gastritis. Diagnosis of Helicobacter pylori infection in gastric mucosa by endoscopic features: a multicenter prospective study. Dig Endosc 2013;25:508-518.

5. Yagi K, Nakamura A, Sekine A. Comparison between magnifying endoscopy and histological, culture and urease test findings from the gastric mucosa of the corpus. Endoscopy 2002;34:376-381.

6. Yagi K, Nakamura A, Sekine A. Characteristic endoscopic and magnified endoscopic findings in the normal stomach without Helicobacter pylori infection. J Gastroenterol Hepatol 2002;17:39-45.

7. Yagi K, Saka A, Nozawa Y, Nakamura A. Prediction of Helicobacter pylori status by conventional endoscopy, narrow-band imaging magnifying endoscopy in stomach after endoscopic resection of gastric cancer. Helicobacter 2014;19:111-115.

8. Tahara T, Shibata T, Nakamura M, et al. Gastric mucosal pattern by using magnifying narrow-band imaging endoscopy clearly distinguishes histological and serological severity of chronic gastritis. Gastrointest Endosc 2009;70:246-253.

9. Kuramoto M, Kubo M. Principles of NBI and BLI-blue laser imaging. In: Tajiri H, Kato M, Tanaka S, Saito Y, editors. NBI/ BLI atlas: new image-enhanced endoscopy. Tokyo, Japan: Nihon Medical Center Inc; 2014, pp. 16-21.

10. Kaneko K, Oono Y, Yano T, et al. Effect of novel bright image enhanced endoscopy using blue laser imaging (BLI). Endosc Int Open 2014;2:E212-E219.

11. Dohi O, Yagi N, Onozawa Y, et al. Linked color imaging improves endoscopic diagnosis of active Helicobacter pylori infection. Endosc Int Open 2016;4:E800-E805.

12. Sonoyama S, Tamaki T, Hirakawa T, et al. Transfer learning for endoscopic image classification. IEEE $J$ Biomed Health Inform 2017;21:41-47.

13. Ribeiro E, Uhl A, Wimmer G, Häfner M. Exploring deep learning and transfer learning for colonic polyp classification. Comput Math Methods Med 2016;2016;6584725.

14. Itoh T, Kawahira H, Nakashima H, Yata N. Deep learning analyzes Helicobacter pylori infection by upper gastrointestinal endoscopy images. Endosc Int Open 2018;6:E139-E144.

15. Shichijo S, Nomura S, Aoyama K, et al. Application of convolutional neural networks in the diagnosis of Helicobacter pylori infection based on endoscopic images. EBioMedicine 2017;25:106-111.

16. Kotachi T, Ito M, Yoshihara M, et al. Serological evaluation of gastric cancer risk based on pepsinogen and Helicobacter pylori antibody: relationship to endoscopic findings. Digestion 2017;95:314-318.

17. Togashi K, Nemoto D, Utano K, et al. Blue laser imaging endoscopy system for the early detection and characterization of colorectal lesions: a guide for the endoscopist. Therap Adv Gastroenterol 2016;9:50-56.

18. Kimura K, Takemoto T. An endoscopic recognition of the atrophic border and its significance in chronic gastritis. Endoscopy 1969;1:87-97.

19. Satoh K, Kimura K, Taniguchi Y, et al. Distribution of inflammation and atrophy in the stomach of Helicobacter pylori-positive and -negative patients with chronic gastritis. Am J Gastroenterol 1996;91:963-969.

20. Lee SY. Current progress toward eradicating Helicobacter pylori in East Asian countries: differences in the 2013 revised guidelines between China, Japan, and South Korea. World J Gastroenterol 2014;20:1493-1502. 
21. Szegedy C, Wei L, Yangqing J, et al. Going deeper with convolutions. In: IEEE conference on computer vision and pattern recognition (CVPR). Boston, MA: IEEE;2015; pp. 1-9.

22. Jia Y, Shelhamer E, Donahue J, et al. Caffe: convolutional architecture for fast feature embedding. In: Proceedings of the $22^{\text {nd }}$ ACM international conference on multimedia. Orlando, FL: ACM; 2014. pp. 675-678.

23. Shin HC, Roth HR, Gao M, et al. Deep convolutional neural networks for computer-aided detection: CNN architectures, dataset characteristics and transfer learning. IEEE Trans Med Imaging 2016;35:1285-1298.

24. Mori Y, Kudo SE, Wakamura K, et al. Novel computer-aided diagnostic system for colorectal lesions by using endocytoscopy (with videos). Gastrointest Endosc 2015;81:621-629.

25. Kanda Y. Investigation of the freely available easy-to-use software 'EZR' for medical statistics. Bone Marrow Transplant
2013;48:452-458.

26. Watanabe K, Nagata N, Shimbo T, et al. Accuracy of endoscopic diagnosis of Helicobacter pylori infection according to level of endoscopic experience and the effect of training. BMC Gastroenterol 2013;13:128.

27. Anagnostopoulos GK, Yao K, Kaye P, et al. High-resolution magnification endoscopy can reliably identify normal gastric mucosa, Helicobacter pylori-associated gastritis, and gastric atrophy. Endoscopy 2007;39:202-207.

28. Xirouchakis E, Laoudi F, Tsartsali L, Spiliadi C, Georgopoulos SD. Screening for gastric premalignant lesions with narrow band imaging, white light and updated Sydney protocol or both? Dig Dis Sci 2013;58:1084-1090.

29. Rugge M, Fassan M, Pizzi M, et al. Autoimmune gastritis: histology phenotype and OLGA staging. Aliment Pharmacol Ther 2012;35:1460-1466. 